\title{
On nonlinear wave equations with parabolic potentials
}

\author{
Alexander I. Komech, ${ }^{1}$ Elena A. Kopylova, ${ }^{2}$ and Sergey A. Kopylov
}

\begin{abstract}
We introduce a new class of piece-wise quadratic potentials for nonlinear wave equations with a kink solutions. The potentials allow an exact description of the spectral properties for the linearized equation at the kink. This description is necessary for the study of the stability properties of the kinks.

In particular, we construct examples of the potentials of Ginzburg-Landau type providing the asymptotic stability of the kinks [6] and [7].
\end{abstract}

Mathematics Subject Classification (2010). 35L70, 37K40.

Keywords. Relativistic invariant nonlinear wave equations, soliton, kink, spectrum, resonance, eigenvalue, Fermi golden rule.

\section{Introduction}

Last two decades there was an outstanding activity in the field of asymptotic stability of solitary waves for nonlinear Schrödinger equations [1], [2], [3], [11], [12], [13], [15], [16], [17], and [18], nonlinear Klein-Gordon equations [5] and [14], relativistic Ginzburg-Landau equations [6] and [7], and other Hamiltonian PDEs [8] and [10]. All these results rely on different assumptions on the spectral properties of the corresponding linearized dynamics. On the other hand, the examples were mostly unknown. Here we construct a model nonlinear wave equations, providing various spectral properties: different number of the eigenvalues, absence of the resonances, and Fermi golden rule.

In particular, we construct the examples of relativistic Ginzburg-Landau equations providing all properties assumed in [6] and [7]. The properties imply the asymptotic stability of kinks for real solutions to 1D nonlinear Ginzburg-Landau equations

$$
\ddot{\psi}(x, t)=\psi^{\prime \prime}(x, t)+F(\psi(x, t)), \quad x \in \mathbb{R},
$$

where $F(\psi)=-U^{\prime}(\psi)$.

We assume the following conditions.

\footnotetext{
${ }^{1}$ Supported partly by Alexander von Humboldt Research Award, the Austrian Science Fund (FWF): P22198-N13 and RFBR grant.

${ }^{2}$ Supported partly by the Austrian Science Fund (FWF): M1329-N13, and RFBR grants.
} 
Condition U1. For some $K>3$ and $m>0$ the potential $U(\psi)$ is smooth even function satisfying

$$
\begin{gathered}
(\psi)>0, \quad \psi \neq a, \\
U(\psi)=\frac{m^{2}}{2}(\psi \mp a)^{2}+\mathcal{O}\left(|\psi \mp a|^{K}\right), \quad \psi \rightarrow \pm a .
\end{gathered}
$$

(see. Figure 1).

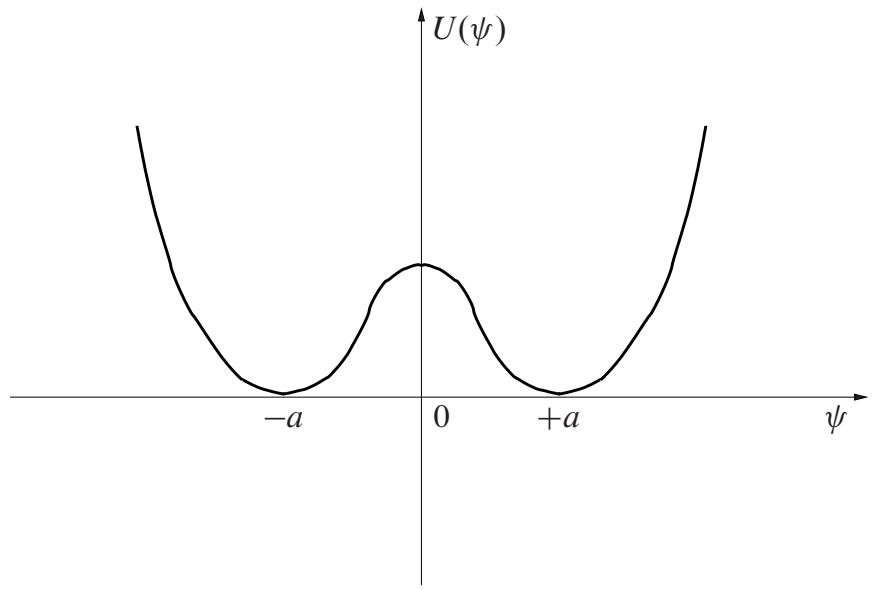

Figure 1. Potential of Ginzburg-Landau type.

The corresponding stationary equation reads

$$
s^{\prime \prime}(x)-U^{\prime}(s(x))=0, \quad x \in \mathbb{R} .
$$

Constant stationary solutions are: $\psi(x) \equiv 0$ and $\psi(x) \equiv \pm a$. There are also the "kinks", i.e. nonconstant finite energy solutions $s(x)$ to (1.2) such that

$$
s(x) \rightarrow \pm a, \quad x \rightarrow \pm \infty .
$$

Condition U1 implies that $(s(x) \mp a)^{\prime \prime} \sim m^{2}(s(x) \mp a)$ for $x \rightarrow \pm \infty$, hence

$$
|s(x) \mp a| \sim C e^{-m|x|}, \quad x \rightarrow \pm \infty \text {. }
$$

(see. Figure 2).

Due to relativistic invariance of equation (1.1) the moving kinks

$$
s_{q, v}(x, t)=s(\kappa(x-v t-q)), \quad q, v \in \mathbb{R},|v|<1, \kappa=1 / \sqrt{1-v^{2}}
$$

also are the solutions to (1.1). Let us linearize equation (1.1) at the kink $s(x)$. Substituting $\psi(x, t)=s(x)+\varphi(x, t)$, we obtain formally

$$
\ddot{\varphi}(x, t)=-H \varphi(x, t)+\mathcal{O}\left(|\varphi(x, t)|^{2}\right),
$$




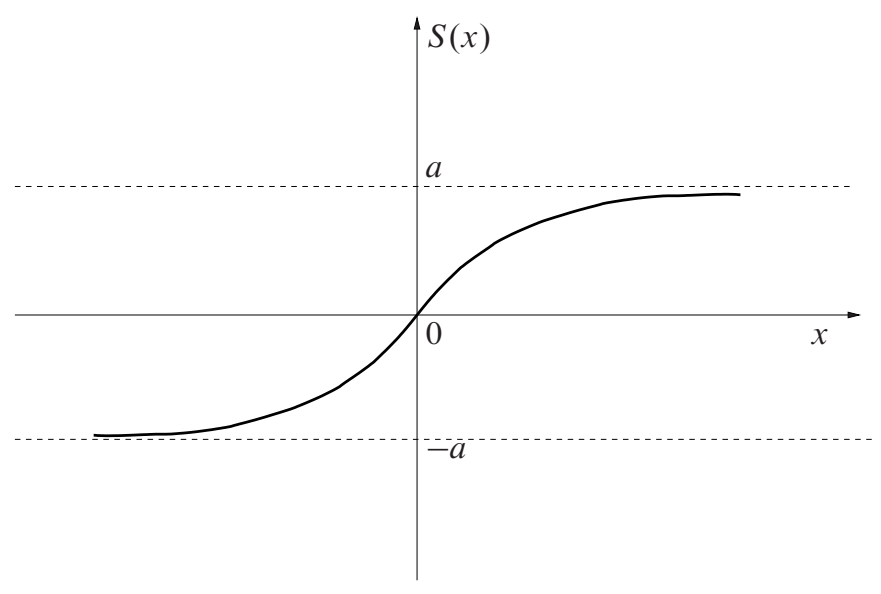

Figure 2. Kink.

where $H$ is the Schrödinger operator

$$
H \stackrel{\text { def }}{=}-\frac{d^{2}}{d x^{2}}+m^{2}+W(x)
$$

with the potential

$$
W(x)=-F^{\prime}(s(x))-m^{2}=U^{\prime \prime}(s(x))-m^{2} .
$$

Condition $\mathbf{U} 1$ and the asymptotics (1.3) imply that

$$
|W(x)|=\mathcal{O}\left(|s(x) \mp a|^{K-1}\right) \sim C e^{-(K-1) m|x|}, \quad x \rightarrow \pm \infty .
$$

The next properties of $H$ hold true.

H1. The continuous spectrum of $H$ is $\sigma_{c}=\left[m^{2}, \infty\right)$.

H2. The point $\lambda_{0}=0$ belongs to the discrete spectrum, and corresponding eigenfunction is $s^{\prime}(x)$.

H3. Since $s^{\prime}(x)>0$, the point $\lambda_{0}=0$ is the groundstate, and all remaining discrete spectrum is contained in $\left(0, m^{2}\right]$.

To establish an asymptotic stability of the kinks $s_{q, v}(x, t)$ one need certain spectral properties of $H$ (cf. [6] and [7]).

Condition U2. The edge point $\lambda=m^{2}$ of the continuous spectrum is neither eigenvalue nor resonance. 
Condition U3. The discrete spectrum of $H$ consists of two points: $\lambda_{0}=0$ and $\lambda_{1} \in\left(0, m^{2}\right)$ satisfying

$$
4 \lambda_{1}>m^{2} \text {. }
$$

We assume also a non-degeneracy condition known as "Fermi golden rule" meaning the strong coupling of the nonlinear term to the continuous spectrum. This coupling provides the energy radiation to infinity (cf. condition (10.0.11) in [2] and condition (1.11) in [7]).

Condition U4. The following inequality holds:

$$
\int \varphi_{4 \lambda_{1}}(x) F^{\prime \prime}(s(x)) \varphi_{\lambda_{1}}^{2}(x) d x \neq 0 .
$$

where $\varphi_{4 \lambda_{1}}$ is the nonzero odd solution to $H \varphi_{4 \lambda_{1}}=4 \lambda_{1} \varphi_{4 \lambda_{1}}$.

Note that the known quartic double well Ginzburg-Landau potential $U_{G L}(\psi)=$ $\left(\psi^{2}-a^{2}\right)^{2} /\left(4 a^{2}\right)$ satisfies condition $\mathbf{U 1}$ with $m^{2}=2$ and $K=3$ as well as conditions $\mathbf{U} \mathbf{3}$ and $\mathbf{U} 4$. However, there exist the resonance for the corresponding operator $H$ at the edge point $\lambda=m^{2}$. Hence, the asymptotic stability of the kinks for $U_{G L}$ is the open problem.

Let us note that the result [4] concerns the wave front solution $\psi\left(x_{1}-v t\right)$ to $3 \mathrm{D}$ wave with the potential $U_{G L}$. The solution has an infinite energy, so it is not a soliton, and its asymptotic stability is provided by the strong dispersion properties of the 3D case. The 1D case requires different arguments [6] and [7].

Our main result is the following theorem.

Theorem 1.1. There exist potentials $U(\psi)$ satisfying conditions $\mathbf{U 1 - U}$ 4.

\section{Piece wise parabolic potentials}

As a first step, we will consider the class of the potentials which are piece-wise second order polynomials.

$$
U_{0}(\psi)= \begin{cases}\frac{1}{2}-\frac{b}{2} \psi^{2}, & |\psi| \leq \gamma, \\ \frac{d}{2}(\psi \mp 1)^{2}, & \pm \psi \geq \gamma,\end{cases}
$$

with some constants $b, d>0$ and $0<\gamma<1$. Let us find the parameters $b=b(\gamma)$ and $d=d(\gamma)$ providing $U_{0}(\psi) \in C^{1}(\mathbb{R})$. We have

$$
U_{0}(\gamma)=\frac{1}{2}-\frac{b}{2} \gamma^{2}=\frac{d}{2}(\gamma-1)^{2} \quad \text { and } \quad U_{0}^{\prime}(\gamma)=-b \gamma=d(\gamma-1) .
$$


Solving the equations, we obtain

$$
b=\frac{1}{\gamma} \quad \text { and } \quad d=\frac{1}{1-\gamma}, \quad 0<\gamma<1 .
$$

Then the functions $U_{0}^{\prime \prime}(\psi)$ are piece-wise constant with the jumps at the points $\psi=$ $\pm \gamma$. Thus, the potentials $U_{0} \in C^{1}(\mathbb{R})$ form one-dimensional manifold parametrized by $\gamma \in(0,1)$.

2.1. Kink. Let us solve the equation of type (1.2) for the kink in the case of potential (2.1):

$$
s_{0}^{\prime \prime}(x)-U_{0}^{\prime}\left(s_{0}(x)\right)=0, \quad x \in \mathbb{R} .
$$

We search an odd solution to

$$
s_{0}^{\prime \prime}(x)= \begin{cases}-b s_{0}(x), & 0<s_{0}(x) \leq \gamma, \\ d\left(s_{0}(x)-1\right), & s_{0}(x)>\gamma .\end{cases}
$$

We have

$$
s_{0}(x)= \begin{cases}C \sin \sqrt{b} x, & 0<x \leq q, \\ A e^{-\sqrt{d} x}+1, & x>q,\end{cases}
$$

where $C>\gamma, A<0, q=\frac{1}{\sqrt{b}} \arcsin \frac{\gamma}{C}$. Equating the values of $s_{0}(x)$ and its left and right derivatives at $x=q$ we obtain

$$
\left\{\begin{array}{l}
A e^{-\sqrt{d} q}+1=C \sin \sqrt{b} q=\gamma \\
-\sqrt{d} A e^{-\sqrt{d} q}=\sqrt{b} C \cos \sqrt{b} q .
\end{array}\right.
$$

The first line of (2.4) implies $A e^{-\sqrt{d} q}=\gamma-1$. Hence the second line of (2.4) becomes

$$
\sqrt{d}(1-\gamma)=\sqrt{b} C \cos \sqrt{b} q .
$$

The both side of the last equality is positive. Hence it is equivalent to

$$
d(1-\gamma)^{2}=b\left(C^{2}-\gamma^{2}\right)
$$

Substituting (2.2) we obtain $1-\gamma=C^{2} / \gamma-\gamma$. Then

$$
C=\sqrt{\gamma}, \quad A=(\gamma-1) e^{\sqrt{\gamma /(1-\gamma)} \arcsin \sqrt{\gamma}}
$$

and

$$
q=\sqrt{\gamma} \arcsin \sqrt{\gamma}
$$


2.2. Linearized equation. Let us linearize equation (1.1) with $F(\psi)=F_{0}(\psi)=$ $-U_{0}(\psi)$ at the kink $s_{0}(x)$ splitting the solution as the sum

$$
\psi(t)=s_{0}+\varphi(t) .
$$

Substituting (2.6) to (1.1), we obtain

$$
\ddot{\varphi}(x, t)=\varphi^{\prime \prime}(x, t)-U_{0}^{\prime}\left(s_{0}(x)+\varphi(x, t)\right)+U_{0}^{\prime}\left(s_{0}(x)\right) .
$$

By (2.1) we can write equations (2.7) as

$$
\ddot{\varphi}(t)=-H_{0} \varphi(t)+\mathcal{N}(\varphi(t)), \quad t \in \mathbb{R},
$$

where

$$
H_{0}=-\frac{d^{2}}{d x^{2}}+W_{0}(x), \quad W_{0}(x)=U_{0}^{\prime \prime}\left(s_{0}(x)\right)= \begin{cases}-b, & |x| \leq q, \\ d, & |x|>q,\end{cases}
$$

(see Figure 3) and

$$
\mathcal{N}(\varphi(t))=-U_{0}^{\prime}\left(s_{0}+\varphi(t)\right)+U_{0}^{\prime}\left(s_{0}\right)+U_{0}^{\prime \prime}\left(s_{0}\right) .
$$

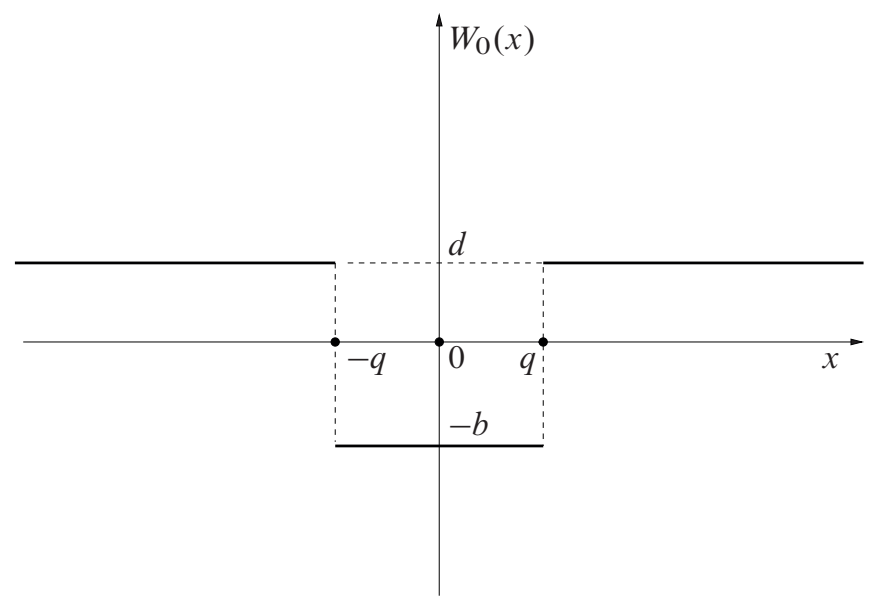

Figure 3. Potential $W_{0}$.

The continuous spectrum of $H_{0}$ coincides with $[d, \infty)$. The point $\lambda_{0}=0$ is the groundstate since it corresponds to the even positive eigenfunction $\varphi_{0}(x)=s_{0}^{\prime}(x)$ :

$$
H_{0} \varphi_{0}=-s_{0}^{\prime \prime \prime}(x)+U_{0}^{\prime \prime}\left(s_{0}(x)\right) s_{0}^{\prime}(x)=0,
$$

which follows by differentiation of (2.3). Therefore, the discrete spectrum of $H_{0}$ belongs to $[0, d]$, and the next eigenfunction $\varphi_{1}(x)$ should be odd. 
2.3. Odd eigenfunctions. Given an eigenvalue $\lambda$, the corresponding eigenfunction $\varphi(x)$ should satisfy the equation

$$
\begin{cases}-\varphi^{\prime \prime}(x)-b \varphi(x)=\lambda \varphi(x), & |x| \leq q, \\ -\varphi^{\prime \prime}(x)+d \varphi(x)=\lambda \varphi(x), & |x|>q .\end{cases}
$$

Equations (2.9) imply that the odd eigenfunctions have the form

$$
\varphi(x)= \begin{cases}B \sin \beta x, & |x| \leq q, \\ A \operatorname{sgn} x e^{-\alpha|x|}, & |x|>q .\end{cases}
$$

where $\alpha=\sqrt{d-\lambda}>0$ and $\beta=\sqrt{b+\lambda}>0$. Equating the values of the eigenfunction and its left and right derivatives at $x=q$, we obtain

$$
\left\{\begin{array}{l}
A e^{-\alpha q}=B \sin \beta q, \\
-A \alpha e^{-\alpha q}=B \beta \cos \beta q,
\end{array}\right.
$$

where $a$ and $b$ are related as follows

$$
\alpha^{2}+\beta^{2}=b+d .
$$

System (2.11) admits nonzero solutions only if its determinant vanishes, that is

$$
-\alpha=\beta \cot \beta q .
$$

At last, multiplying by $q$, and denoting $\xi=\beta q$ and $\eta=\alpha q$, we obtain the system of equations

$$
-\eta=\xi \cot \xi, \quad \xi^{2}+\eta^{2}=R^{2}
$$

where $R=q \sqrt{b+d}$ is the radius of the circle. Substituting $b, d$ and $q$ from (2.2) and (2.5) respectively, we obtain

$$
R=q \sqrt{\frac{1}{\gamma}+\frac{1}{1-\gamma}}=\frac{q}{\sqrt{\gamma(1-\gamma)}}=\frac{\arcsin \sqrt{\gamma}}{\sqrt{1-\gamma}} .
$$

Finally, the solutions to (2.12) can be found graphically (see Figure 4). Taking into account that $\eta>0$, we obtain that

$$
\begin{gathered}
R \in\left(0, \frac{\pi}{2}\right]: \text { system (2.12) has no solution; } \\
R \in\left(\frac{\pi}{2}, \frac{3 \pi}{2}\right]: \text { system (2.12) has one solution; } \\
R \in\left(\frac{3 \pi}{2}, \frac{5 \pi}{2}\right]: \text { system (2.12) has two solutions; }
\end{gathered}
$$




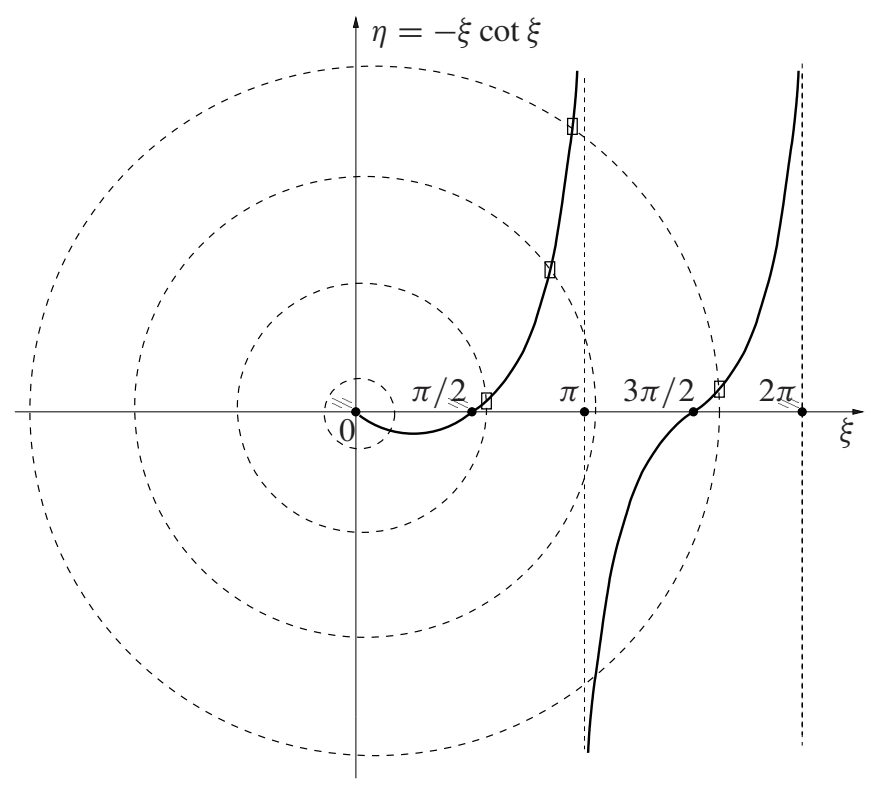

Figure 4. Graphical solution of system (2.12).

Let us note that $R(0)=0$ and $R(1)=\infty$, and the radius $R(\gamma)$ is monotone increasing on $[0,1]$. Denote by $\gamma_{k}, k \in \mathbb{N}$ the solution to the equation

$$
\frac{\arcsin \sqrt{\gamma_{k}}}{\sqrt{1-\gamma_{k}}}=\frac{k \pi}{2}, \quad k \in \mathbb{N} .
$$

Numerical calculations give

$$
\gamma_{1} \sim 0.64643, \quad \gamma_{2} \sim 0.8579, \quad \gamma_{3} \sim 0.92472, \quad \gamma_{4} \sim 0.95359 \ldots
$$

Further, (2.13) implies that

$\gamma \in\left(0, \gamma_{1}\right]:$ no nonzero odd eigenfunctions,

$\gamma \in\left(\gamma_{1}, \gamma_{3}\right]$ : one linearly independent odd eigenfunction,

$\gamma \in\left(\gamma_{3}, \gamma_{5}\right]$ : two linearly independent odd eigenfunctions, 
In particular, for $\gamma \in\left(\gamma_{1}, \gamma_{3}\right]$ we have one odd eigenfunction and the corresponding eigenvalue $\lambda_{1} \in(0, d)$ reads

$$
\begin{aligned}
\lambda_{1} & =\lambda_{1}(\gamma)=\beta^{2}-b=\frac{\xi^{2}}{q^{2}}-b \\
& =\frac{1}{\gamma}\left(\frac{\xi^{2}}{\arcsin ^{2} \sqrt{\gamma}}-1\right)=\frac{1}{\gamma}\left(\frac{\sin ^{2} \xi}{1-\gamma}-1\right),
\end{aligned}
$$

where $\xi$ is the solution to

$$
\frac{\xi^{2}}{\sin ^{2} \xi}=\frac{\arcsin ^{2} \sqrt{\gamma}}{1-\gamma}
$$

2.4. Even eigenfunctions. Equations (2.9) imply that the even eigenfunctions have the form

$$
\varphi(x)= \begin{cases}B \cos \beta x, & |x| \leq q, \\ A e^{-\alpha|x|}, & |x|>q,\end{cases}
$$

where $\alpha=\sqrt{d-\lambda}>0, \beta=\sqrt{b+\lambda}>0$. Equating the values of the eigenfunction and its left and right derivatives at $x=q$, we obtain

$$
\left\{\begin{array}{l}
A e^{-\alpha q}=B \cos \beta q, \\
A \alpha e^{-\alpha q}=B \beta \sin \beta q .
\end{array}\right.
$$

The system admits nonzero solutions if and only if its determinant vanishes:

$$
\alpha=\beta \tan \beta q
$$

Similarly (2.12), we obtain the following equations for $\xi=\beta q$ and $\eta=\alpha q$ :

$$
\eta=\xi \tan \xi, \quad \xi^{2}+\eta^{2}=R^{2}
$$

where $R=\frac{\arcsin \sqrt{\gamma}}{\sqrt{1-\gamma}}$. The solutions can also be found graphically (see Figure 5). 


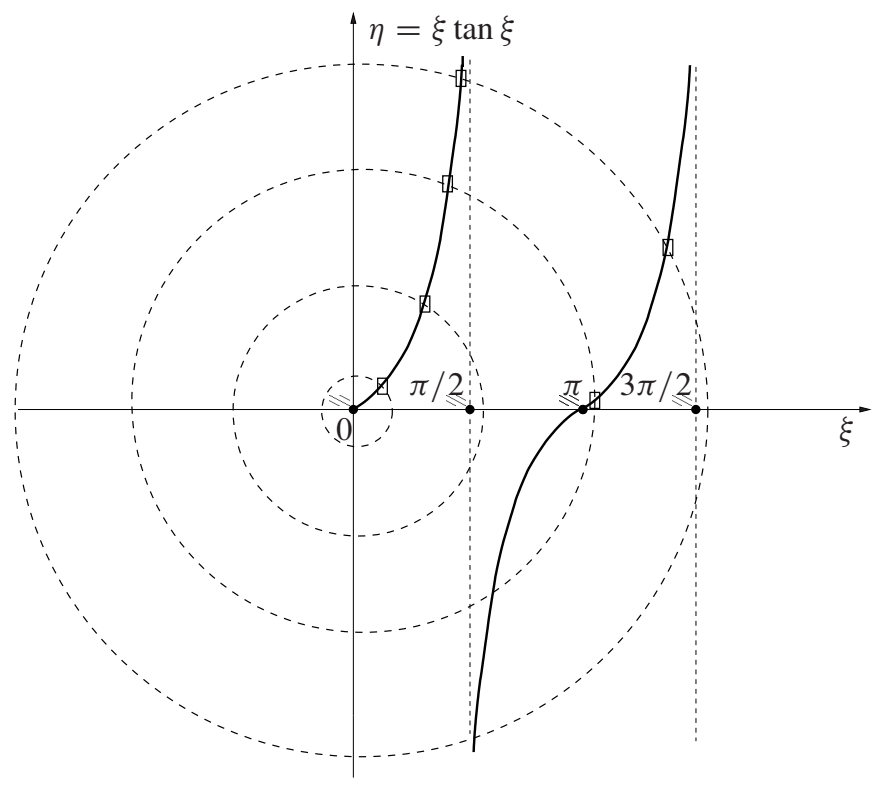

Figure 5. Graphical solution of system (2.19).

We have

$$
\begin{aligned}
& R \in(0, \pi]: \text { system }(2.19) \text { has one solution; } \\
& R \in(\pi, 2 \pi]: \text { system }(2.19) \text { has two solutions; }
\end{aligned}
$$

Note that for any $\gamma \in(0,1)$ equation (2.19) has the solution $\xi=\arcsin \sqrt{\gamma} \in$ $(0, \pi / 2)$. The solution corresponds to eigenvalue $\lambda=0$ and the first even eigenfunction. Moreover, (2.20) implies that

$$
\begin{aligned}
& \gamma \in\left(0, \gamma_{2}\right]: \text { one linearly independent even eigenfunction, } \\
& \gamma \in\left(\gamma_{2}, \gamma_{4}\right]: \text { two linearly independent even eigenfunctions, }
\end{aligned}
$$

where $\gamma_{i}$ are defined in (2.14).

Conclusion. 1) There is one eigenvalue $\lambda_{0}=0$ for $\gamma \in\left(0, \gamma_{1}\right]$. 2) There are two eigenvalues $\lambda_{0}=0$ and $0<\lambda_{1}<d$ for $\gamma \in\left(\gamma_{1}, \gamma_{2}\right.$. Etc. (See Figure 6.) 


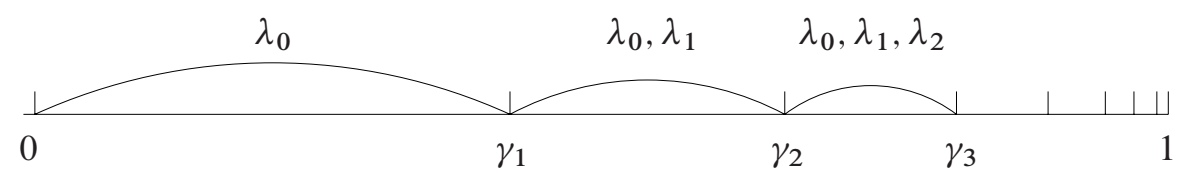

Figure 6. Spectrum.

\section{Spectral conditions}

We deduce Theorem 1.1 in Section 4 below from the following proposition.

Proposition 3.1. For any $\gamma \in\left(\gamma_{1}, \gamma_{2}\right)$ the piece wise parabolic potentials $U_{0}$, defined

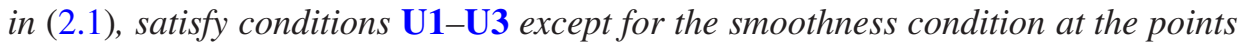
$\psi= \pm \gamma$. Condition $\mathbf{U} 4$ holds for any $\gamma \in\left(\gamma_{1}, \gamma_{2}\right)$ except for one point $\gamma_{*}$.

Proof. Step i) Obviously, for $U_{0}(\psi)$ condition U1 with $a=1, m^{2}=d$, and any integer $K \geq 3$ holds except the smoothness at the points $\psi= \pm \gamma$.

Consider condition U2. Note that the solutions to (2.12) or (2.19) with $\eta=0$ and $R=k \pi / 2, k \in \mathbb{N}$ correspond to $\alpha=0$ i.e. $\lambda=d$. Then the functions (2.10) or (2.18) with $A \neq 0$ are a nonzero constant for $|x| \geq \gamma$. Hence, the functions are the resonances corresponding to the edge point $\lambda=d$ of the continuous spectrum. Thus, the resonances exist only for the discrete set of parameters $\gamma_{k}, k \in \mathbb{N}$, defined in (2.14). Evidently, the set has just one limit point 1. Hence, condition U2 holds for $\gamma \in(0,1) \backslash\left\{\cup_{k \in \mathbb{N}} \gamma_{k}\right\}$.

Step ii) For any $\gamma \in\left(\gamma_{1}, \gamma_{2}\right)$ the operator $H_{0}$ defined in (2.8) has exactly two eigenvalues $\lambda_{0}=0$ and $\lambda_{1} \in(0, d)$. For condition $\mathbf{U} 3$ it remains to verify (1.4) with $m^{2}=d$. Namely, due to (2.16) and (2.17) we must prove that for any $\gamma \in\left(\gamma_{1}, \gamma_{2}\right)$ the following inequality holds:

$$
\frac{4}{\gamma}\left(\frac{\sin ^{2} \xi(\gamma)}{1-\gamma}-1\right)>\frac{1}{1-\gamma}
$$

where $\xi(\gamma) \in(\pi / 2, \pi)$ is the solution to (2.17). After the simple transformations we obtain

$$
4 \cos ^{2} \xi(\gamma)<3 \gamma,
$$

and

$$
\frac{\pi}{2}<\xi(\gamma)<\pi-\arccos \frac{\sqrt{3 \gamma}}{2} .
$$

Since $\frac{\xi}{\sin \xi}$ is monotonically increasing function for $\xi \in(\pi / 2, \pi)$, then

$$
\frac{\pi}{2}<\frac{\arcsin \sqrt{\gamma}}{\sqrt{1-\gamma}}<\frac{2\left(\pi-\arccos \frac{\sqrt{3 \gamma}}{2}\right)}{\sqrt{4-3 \gamma}}
$$


Finally, we obtain

$$
\gamma_{1}<\gamma<\alpha
$$

where $\alpha$ is the solution to

$$
\frac{\arcsin \sqrt{\alpha}}{\sqrt{1-\alpha}}=\frac{2\left(\pi-\arccos \frac{\sqrt{3 \alpha}}{2}\right)}{\sqrt{4-3 \alpha}} .
$$

Numerical calculation gives

$$
\alpha=0.921485>\gamma_{2} .
$$

Therefore, condition $\mathbf{U} 3$ holds for any $\gamma \in\left(\gamma_{1}, \gamma_{2}\right)$.

Step iii) Finally, consider condition U4 (Fermi golden rule). The condition can be rewritten as

$$
\int U_{0}^{\prime \prime \prime}\left(s_{0}(x)\right) \varphi_{4 \lambda_{1}}(x) \varphi_{\lambda_{1}}^{2}(x) d x=\int \frac{d}{d x} U_{0}^{\prime \prime}\left(s_{0}(x)\right) \frac{\varphi_{4 \lambda_{1}}(x) \varphi_{\lambda_{1}}^{2}(x)}{s_{0}^{\prime}(x)} d x \neq 0 .
$$

By (2.8) we have that $U_{0}^{\prime \prime}\left(s_{0}(x)\right)=W_{0}(x)$ is the piece wise constant function. Hence,

$$
\frac{d}{d x} U_{0}^{\prime \prime}\left(s_{0}(x)\right)=(b+d) \delta(x-q)-(b+d) \delta(x+q),
$$

and (3.1) becomes

$$
\varphi_{4 \lambda_{1}}(q) \varphi_{\lambda_{1}}^{2}(q) \neq 0 \text {. }
$$

Formula (2.10) yields that $\varphi_{\lambda_{1}}(q)=A e^{-\alpha q} \neq 0$. Hence it is sufficient to verify that

$$
\varphi_{4 \lambda_{1}}(q) \neq 0 .
$$

The eigenfunction $\varphi_{4 \lambda_{1}}$ satisfies the equations

$$
\begin{cases}-\varphi_{4 \lambda_{1}}^{\prime \prime}(x)-b \varphi_{4 \lambda_{1}}(x)=4 \lambda_{1} \varphi_{4 \lambda_{1}}(x), & |x| \leq q, \\ -\varphi_{4 \lambda_{1}}^{\prime \prime}(x)+d \varphi_{4 \lambda_{1}}(x)=4 \lambda_{1} \varphi_{4 \lambda_{1}}(x), & |x|>q .\end{cases}
$$

For the odd solution to (3.2) we have

$$
\varphi_{4 \lambda_{1}}(q)=\sin \beta q, \quad \beta=\sqrt{b+4 \lambda_{1}}>0 .
$$

Therefore, $\varphi_{4 \lambda_{1}}(q)=0$ only if $\beta q=k \pi, k \in \mathbb{N}$, or

$$
\sqrt{1+4 \gamma \lambda_{1}(\gamma)} \arcsin \sqrt{\gamma}=k \pi, \quad k \in \mathbb{N},
$$

where $\lambda_{1}(\gamma)$ is defined in (2.16) and (2.17). Substituting $\lambda_{1}(\gamma)$ into (3.3) we obtain from (2.16) and (2.17)

$$
\left\{\begin{array}{l}
\frac{\arcsin \sqrt{\gamma}}{\sqrt{1-\gamma}} \sqrt{4 \sin ^{2} \xi-3(1-\gamma)}=k \pi, \\
\frac{\xi^{2}}{\sin ^{2} \xi}=\frac{\arcsin ^{2} \sqrt{\gamma}}{1-\gamma} .
\end{array}\right.
$$


For $\gamma \in\left(\gamma_{1}, \gamma_{2}\right)$ the system has a solution only for $k=1$ since

$$
0<\frac{\arcsin \sqrt{\gamma}}{\sqrt{1-\gamma}} \sqrt{4 \sin ^{2} \xi-3(1-\gamma)}<2 \pi, \quad \gamma_{1}<\gamma<\gamma_{2} .
$$

Denote

$$
\theta=\arcsin \sqrt{\gamma} \in\left(\pi \sqrt{1-\gamma_{1}} / 2, \pi \sqrt{1-\gamma_{2}}\right) .
$$

Then (3.4) with $k=1$ is equivalent to

$$
\left\{\begin{array}{l}
4 \xi^{2}-3 \theta^{2}=\pi^{2} \\
\frac{\sin \xi}{\xi}=\frac{\cos \theta}{\theta}
\end{array}\right.
$$

Let us prove that (3.6) has a unique solution. Consider two functions $\theta_{1}(\xi)$ and $\theta_{2}(\xi)$, where $\theta_{1}(\xi) \stackrel{\text { def }}{=} \frac{1}{\sqrt{3}} \sqrt{4 \xi^{2}-\pi^{2}}$ and $\theta_{2}(\xi)$ is the solution of $\frac{\sin \xi}{\xi}=\frac{\cos \theta}{\theta}$. The function $\theta_{1}(\xi)$ increases for $\xi\left(\gamma_{1}\right)<\xi<\xi\left(\gamma_{2}\right)$, and

$$
\theta_{1}^{\prime}(\xi)=\frac{1}{\sqrt{3}} \frac{4 \xi}{\sqrt{4 \xi^{2}-\pi^{2}}}>\frac{1}{\sqrt{3}} \frac{4(\pi / 2)}{\sqrt{4(3 \pi / 4)^{2}-\pi^{2}}}=\frac{4}{\sqrt{15}}>1
$$

for $\xi\left(\gamma_{2}\right)<\xi<\xi\left(\gamma_{2}\right)$, since $\xi\left(\gamma_{1}\right)=\pi / 2$ and $\xi\left(\gamma_{2}\right) \sim 2.3137<3 \pi / 4$. On the other hand,

$$
\theta_{2}^{\prime}(\xi)=\frac{\sin \xi-\xi \cos \xi}{\xi^{2}} \frac{\theta^{2}}{\cos \theta+\theta \sin \theta}>0, \quad \pi / 2<\xi<\xi\left(\gamma_{2}\right)
$$

Moreover, by (3.5) and (3.6) we obtain

$$
\theta_{2}^{\prime}(\xi)=\frac{\theta}{\xi} \frac{\frac{\sin \xi}{\xi}-\cos \xi}{\frac{\cos \theta}{\theta}+\sin \theta}<\frac{\frac{\sin \xi}{\xi}-\cos \xi}{\frac{\sin \xi}{\xi}+\sin \theta}<1, \quad \pi / 2<\xi<\xi\left(\gamma_{2}\right)
$$

since $|\cos \xi|<\left|\cos \xi\left(\gamma_{2}\right)\right|<\sqrt{2} / 2$, and $\sin \theta=\sqrt{\gamma}>\sqrt{\gamma}_{1}>\sqrt{2} / 2$ by (2.15). Finally,

$$
\theta_{2}(\pi / 2)>\theta_{1}(\pi / 2)=0, \quad \theta_{2}\left(\xi\left(\gamma_{2}\right)\right) \sim 1.1843<\theta_{1}\left(\xi\left(\gamma_{2}\right)\right) \sim 1.9616 .
$$

Therefore, (3.7) and (3.8) imply that $\theta_{1}(\xi)=\theta_{2}(\xi)$ for a single value $\xi\left(\gamma_{*}\right) \in$ $\left(\pi / 2, \xi\left(\gamma_{2}\right)\right)$ (see Figure 7). 


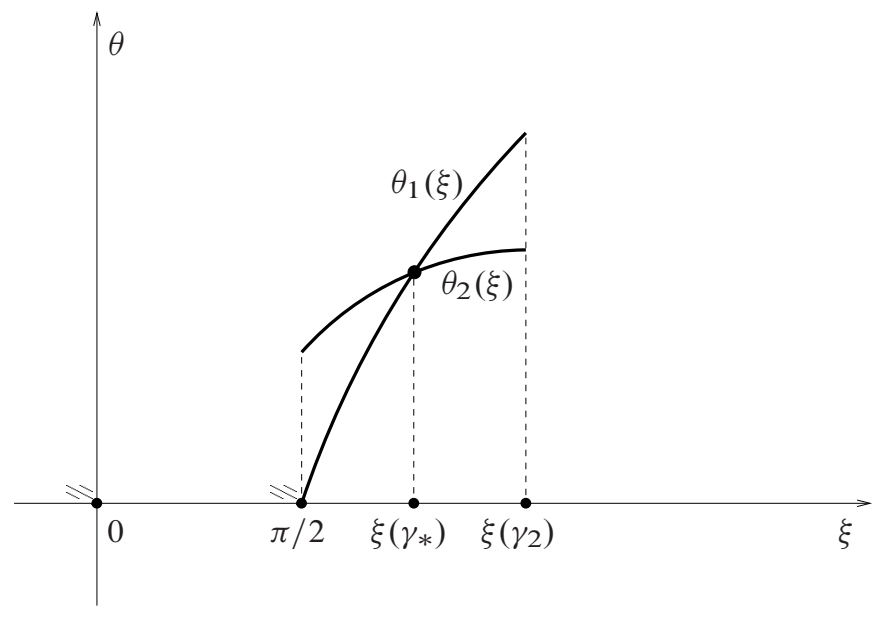

Figure 7. Functions $\theta_{1}$ and $\theta_{2}$.

Numerical calculation gives $\gamma_{*} \sim 0.7925$. Hence, system (3.6) on the interval $\left(\gamma_{1}, \gamma_{2}\right)$ has the only solution $\gamma=\gamma_{*}$. Thus, the Fermi golden rule holds for any $\gamma \in\left(\gamma_{1}, \gamma_{2}\right)$ except for the one point $\gamma_{*}$.

Conclusion. The potential $U_{0}(\psi)$ satisfies conditions $\mathbf{U 1}-\mathbf{U} 4$ except for the smoothness at the points $\psi= \pm \gamma$ for any $\gamma \in\left(\gamma_{1}, \gamma_{*}\right) \cup\left(\gamma_{*}, \gamma_{2}\right)$.

\section{Smooth potentials}

We deduce Theorem 1.1 from Proposition 3.1 by an approximation of the potential $U_{0}$ with a smooth functions satisfying conditions U1-U4. Namely, let $h(\psi) \in C_{0}^{\infty}(\mathbb{R})$ be an even mollifying function with the following properties:

$$
h(\psi) \geq 0, \quad \operatorname{supp} h \subset[-1,1], \quad \int h(\psi) d \psi=1 .
$$

For $\varepsilon \in(0,1]$ we set

$$
\tilde{U}_{\varepsilon}(\psi) \stackrel{\text { def }}{=} \frac{1}{\varepsilon} \int h\left(\frac{\psi-\psi^{\prime}}{\varepsilon}\right) U_{0}\left(\psi^{\prime}\right) d \psi^{\prime} .
$$

Evidently, $\tilde{U}_{\varepsilon}(\psi) \geq 0$ is a smooth, even function, symmetric with respect to the points $\psi= \pm 1$ in some neighborhoods of these points. In addition we have

$$
\tilde{U}_{\varepsilon}(\psi)-U_{0}(\psi)= \begin{cases}\mu_{\varepsilon}>0, & |\psi| \geq \gamma+\varepsilon \\ -v_{\varepsilon}<0, & |\psi| \leq \gamma-\varepsilon\end{cases}
$$


where $\mu_{\varepsilon}, v_{\varepsilon}=\mathcal{O}\left(\varepsilon^{2}\right)$. Let us set

$$
U_{\varepsilon}(\psi)=\tilde{U}_{\varepsilon}(\psi)-\mu_{\varepsilon}
$$

Then

$$
U_{\varepsilon}(\psi)= \begin{cases}U_{0}(\psi), & |\psi| \geq \gamma+\varepsilon \\ U_{0}(\psi)-\mu_{\varepsilon}-v_{\varepsilon}, & |\psi| \leq \gamma-\varepsilon\end{cases}
$$

Obviously,

$$
\sup _{\psi \in \mathbb{R}}\left|U_{\varepsilon}(\psi)-U_{0}(\psi)\right| \leq C \varepsilon
$$

with some constant $C$. Moreover,

$$
U_{\varepsilon}^{\prime \prime \prime}(\psi) \leq 0 \text { for } \psi \leq 0 \text { and } U_{\varepsilon}^{\prime \prime \prime}(\psi) \geq 0 \text { for } \psi \geq 0 .
$$

The corresponding kink is an odd solution to the equation

$$
s_{\varepsilon}^{\prime \prime}(x)-U_{\varepsilon}^{\prime}\left(s_{\varepsilon}(x)\right)=0, \quad x \in \mathbb{R} .
$$

The equation can be integrated using the "energy conservation"

$$
\frac{\left|s_{\varepsilon}^{\prime}(x)\right|^{2}}{2}-U_{\varepsilon}\left(s_{\varepsilon}(x)\right)=\text { const }, \quad x \in \mathbb{R}
$$

with const $=0$ :

$$
\int_{0}^{s_{\varepsilon}(x)} \frac{d s}{\sqrt{2 U_{\varepsilon}(s)}}=x, \quad x \in \mathbb{R} .
$$

Hence, $s_{\varepsilon}(x)$ is a monotone increasing function, and

$$
s_{\varepsilon}(x) \rightarrow \pm 1, \quad x \rightarrow \pm \infty .
$$

Moreover, (4.3), (4.4), and (4.6) imply that

$$
\sup _{x \in \mathbb{R}}\left|s_{\varepsilon}(x)-s_{0}(x)\right| \leq C_{1} \varepsilon .
$$

Therefore,

$$
|| s_{\varepsilon}(x)|-\gamma| \geq \varepsilon, \quad|| x|-q| \geq \delta,
$$

where

$$
\delta \longrightarrow 0 \quad \text { as } \varepsilon \rightarrow 0 .
$$

Hence, for the linear potential $W_{\varepsilon}(x) \stackrel{\text { def }}{=} U_{\varepsilon}^{\prime \prime}\left(s_{\varepsilon}(x)\right)$ we obtain

$$
W_{\varepsilon}(x)=W_{0}(x), \quad|| x|-q| \geq \delta .
$$

Further, (4.5) implies that

$$
W_{\varepsilon}^{\prime}(x) \leq 0 \text { for } x \leq 0 \quad \text { and } \quad W_{\varepsilon}^{\prime}(x) \geq 0 \text { for } x \geq 0 .
$$


Therefore,

$$
\left|W_{\varepsilon}(x)-W_{0}(x)\right| \leq b+d, \quad x \in \mathbb{R} .
$$

(see Figure 8).

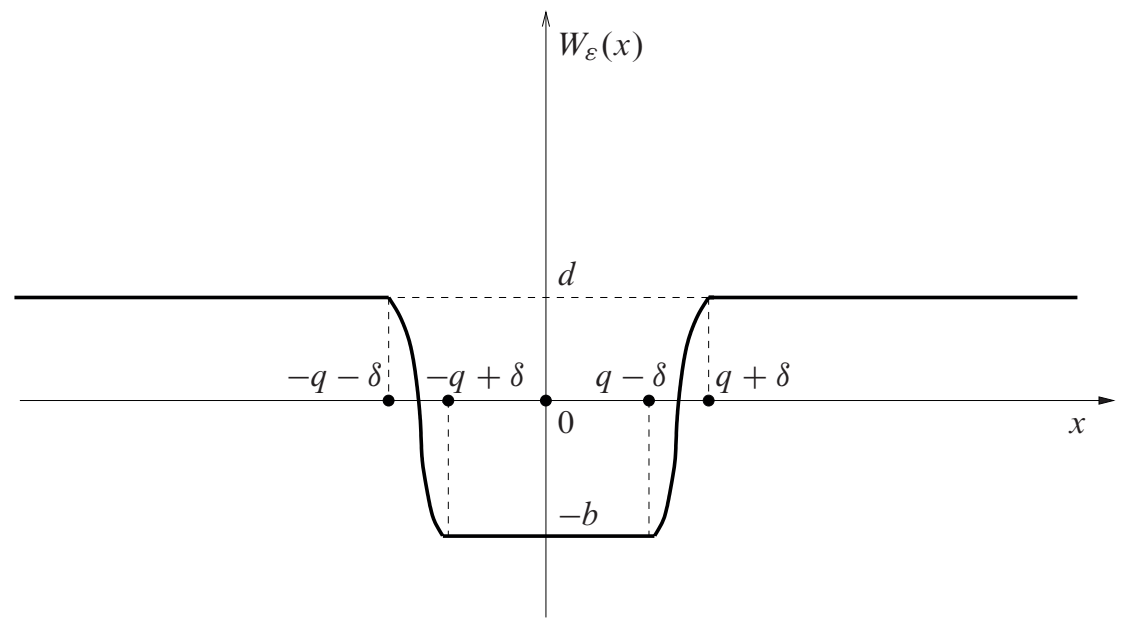

Figure 8. Potential $W_{\varepsilon}$.

As a result, denoting $w_{\varepsilon}(x)=W_{\varepsilon}(x)-W_{0}(x)$, we obtain

$$
\left\|w_{\varepsilon}\right\|_{L^{2}(\mathbb{R})} \longrightarrow 0, \quad \varepsilon \rightarrow 0
$$

by (4.7), (4.8), and (4.9).

Lemma 4.1. The eigenvalues of the Schrödinger operator

$$
H_{\varepsilon}=-\frac{d^{2}}{d x^{2}}+W_{\varepsilon}(x)
$$

converge to the ones of $H_{0}$ as $\varepsilon \rightarrow 0$.

Proof. The eigenvalues of $H_{0}$ and $H_{\varepsilon}$ are the poles of the resolvents $R_{0}(\omega)=$ $\left(H_{0}-\omega\right)^{-1}$ and $R_{\varepsilon}(\omega)=\left(H_{\varepsilon}-\omega\right)^{-1}$ respectively. Hence, the lemma follows from (4.10) due to the relation

$$
R_{\varepsilon}(\omega)=\left(H_{0}-\omega+w_{\varepsilon}\right)^{-1}=R_{0}(\omega)\left(1+w_{\varepsilon} R_{0}(\omega)\right)^{-1} .
$$

Proof of Theorem 1.1. Consider the potential $U(\psi)=U_{\varepsilon}(\psi)$ defined in eq. (4.1) and eq. (4.2). Let us prove that there exist $\varepsilon_{0}>0$ such that for any $\gamma \in\left(\gamma_{1}, \gamma_{2}\right) \backslash \gamma_{*}$, and $0<\varepsilon<\varepsilon_{0}$ the potential $U_{\varepsilon}$ satisfies conditions $\mathbf{U 1}-\mathbf{U} 4$. 
Step i) Condition $\mathbf{U} \mathbf{1}$ with $a=1, m^{2}=d$, and any integer $K \geq 3$ obviously holds.

Step ii) For any $\sigma \in \mathbb{R}$, and $s=0,1,2, \ldots$ denote by $\mathscr{H}_{\sigma}^{s}=\mathscr{H}_{\sigma}^{s}(\mathbb{R})$ the weighted Sobolev spaces with the finite norms

$$
\|\psi\|_{\mathscr{H}_{\sigma}^{s}}=\sum_{k=0}^{s}\left\|(1+|x|)^{\sigma} \psi^{(k)}\right\|_{L^{2}(\mathbb{R})}<\infty .
$$

By [9], Theorem 7.2, the absence of the resonance at the point $\omega=d$ for the Schrödinger operator $H$ is equivalent to the boundedness of the corresponding resolvent $R(\omega): \mathscr{H}_{\sigma}^{0} \rightarrow \mathscr{H}_{-\sigma}^{2}$ at $\omega=d$ for any $\sigma>1 / 2$. Hence, the resolvent $R_{0}(d): \mathscr{H}_{\sigma}^{0} \rightarrow \mathscr{H}_{-\sigma}^{2}$ is bounded by Proposition 3.1. Further, (4.8) and (4.9) imply

$$
\left\|w_{\varepsilon}\right\|_{\mathscr{H}_{-\sigma}^{0} \rightarrow H_{\sigma}^{0}} \longrightarrow 0, \quad \varepsilon \rightarrow 0
$$

Hence, for sufficiently small $\varepsilon$ the operator $R_{\varepsilon}(d): \mathscr{H}_{\sigma}^{0} \rightarrow \mathscr{H}_{-\sigma}^{2}$ is bounded by (4.11). Then condition $\mathbf{U} \mathbf{2}$ holds for $U_{\varepsilon}$.

Step iii) Lemma 4.1 implies that for $\gamma \in\left(\gamma_{1}, \gamma_{2}\right)$ and sufficiently small $\varepsilon$ the operator $H_{\varepsilon}$ has exactly two eigenvalues $\lambda_{0}=0$ and $0<\lambda_{1}(\varepsilon)<d$. Moreover, $\lambda_{1}(\varepsilon) \rightarrow \lambda_{1}(0)=\lambda_{1}$ as $\varepsilon \rightarrow 0$ and then $4 \lambda_{1}(\varepsilon)>d$ for sufficiently small $\varepsilon$. Hence, condition $\mathbf{U} \mathbf{3}$ holds.

Step iv) It remains to check condition U4. Consider arbitrary $\gamma \in\left(\gamma_{1}, \gamma_{2}\right) \backslash \gamma_{*}$. Denote $\varphi_{\lambda_{1}(\varepsilon)}^{\varepsilon}$ and $\varphi_{4 \lambda_{1}(\varepsilon)}^{\varepsilon}$ the corresponding odd eigenfunctions of $H_{\varepsilon}$. Then we have

$$
\begin{aligned}
& \int U_{\varepsilon}^{\prime \prime \prime}\left(s_{\varepsilon}(x)\right) \varphi_{4 \lambda_{1}(\varepsilon)}^{\varepsilon}(x)\left(\varphi_{\lambda_{1}(\varepsilon)}^{\varepsilon}(x)\right)^{2} d x \\
& =\int_{|x-q| \leq \delta} \frac{d}{d x} W_{\varepsilon}(x) \frac{\varphi_{4 \lambda_{1}(\varepsilon)}^{\varepsilon}(x)\left(\varphi_{\lambda_{1}(\varepsilon)}^{\varepsilon}(x)\right)^{2}}{s_{\varepsilon}^{\prime}(x)} d x \\
& =\sum_{ \pm} d \frac{\varphi_{4 \lambda_{1}(\varepsilon)}^{\varepsilon}( \pm q+\delta)\left(\varphi_{\lambda_{1}(\varepsilon)}^{\varepsilon}( \pm q+\delta)\right)^{2}}{s_{\varepsilon}^{\prime}( \pm q+\delta)} \\
& \quad+\sum_{ \pm} b \frac{\varphi_{4 \lambda_{1}(\varepsilon)}^{\varepsilon}( \pm q-\delta)\left(\varphi_{\lambda_{1}(\varepsilon)}^{\varepsilon}( \pm q-\delta)\right)^{2}}{s_{\varepsilon}^{\prime}( \pm q-\delta)} \\
& \quad-\int_{|x-q| \leq \delta} W_{\varepsilon}(x) \frac{d}{d x} \frac{\varphi_{4 \lambda_{1}^{\varepsilon}(\varepsilon)}^{\varepsilon}(x)\left(\varphi_{\lambda_{1}(\varepsilon)}^{\varepsilon}(x)\right)^{2}}{s_{\varepsilon}^{\prime}(x)} d x \\
& \underset{\varepsilon \rightarrow 0}{\longrightarrow} 2(d+b) \frac{\varphi_{4 \lambda_{1}}(q) \varphi_{\lambda_{1}}^{2}(q)}{s_{0}^{\prime}(q)}=\int U_{0}^{\prime \prime \prime}\left(s_{0}(x)\right) \varphi_{4 \lambda_{1}}(x) \varphi_{\lambda_{1}}^{2}(x) d x \neq 0
\end{aligned}
$$

since $\delta \rightarrow 0$ as $\varepsilon \rightarrow 0$. Hence, $\mathbf{U} 4$ holds for sufficiently small $\varepsilon$. 


\section{References}

[1] V. Buslaev and G. Perelman, On the stability of solitary waves for nonlinear Schrödinger equations. In N. N. Uraltseva (ed), Nonlinear evolution equations. Translation edited by A. B. Sossinsky. American Mathematical Society Translations, Series 2, 164. Advances in the Mathematical Sciences 22. Amer. Math. Soc., Providence, R.I., 1995, 75-98. MR 1334139 Zbl 0841.35108

[2] V. Buslaev and C. Sulem, On asymptotic stability of solitary waves for nonlinear Schrödinger equations. Ann. Inst. Henri Poincaré Anal. Non Linéaire 20 (2003), 419-475. MR 1972870 Zbl 1028.35139

[3] S. Cuccagna, The Hamiltonian structure of the nonlinear Schrödinger equation and the asymptotic stability of its ground states. Comm. Math. Phys. 305 (2011), 279-331. MR 2805462 Zbl 1222.35183

[4] S. Cuccagna, On asymptotic stability in 3D of kinks for the $\varphi^{4}$ model. Trans. Amer. Math. Soc. 360 (2008), 2581-2614. MR 2373326 Zbl 1138.35062

[5] V. Imaikin, A. Komech, and B. Vainberg, On scattering of solitons for the Klein-Gordon equation coupled to a particle. Comm. Math. Phys. 268 (2006), 321-367. MR 2259198 Zbl 1127.35054

[6] E. Kopylova and A. Komech, On asymptotic stability of moving kink for relativistic Ginzburg-Landau equation. Comm. Math. Phys. 302 (2011), 225-252. MR 2770013 Zbl 1209.35134

[7] E. Kopylova and A. Komech, On asymptotic stability of kink for relativistic Ginzburg-Landau equation. Arch. Rat. Mech. Anal. 202 (2011), 213-245. MR 2835867 Zbl 1209.35134

[8] J. Miller and M. Weinstein, Asymptotic stability of solitary waves for the regularized long-wave equation. Comm. Pure Appl. Math. 49 (1996), 399-441. MR 1376657 Zbl 0854.35102

[9] M. Murata, Asymptotic expansions in time for solutions of Schrödinger-type equations. J. Funct. Anal. 49 (1982), 10-56. MR 0680855 Zbl 0499.35019

[10] R. Pego and M. I. Weinstein, Asymptotic stability of solitary waves. Comm. Math. Phys. 164 (1994), 305-349. MR 1289328 Zbl 0805.35117

[11] I. Sigal, Nonlinear wave and Schrödinger equations. I. Instability of periodic and quasiperiodic solutions. Comm. Math. Phys. 153 (1993), 297-320. MR 1218303 Zbl 0780.35106

[12] A. Soffer and M. I. Weinstein, Multichannel nonlinear scattering for nonintegrable equations. Comm. Math. Phys. 133 (1990), 119-146. MR 1071238 Zbl 0721.35082

[13] A. Soffer and M. I. Weinstein, Multichannel nonlinear scattering for nonintegrable equations. II. The case of anisotropic potentials and data. J. Diff. Equations 98 (1992), 376-390. MR 1170476 Zbl 0795.35073

[14] A. Soffer and M. I. Weinstein, Resonances, radiation damping and instability in Hamiltonian nonlinear wave equations. Invent. Math. 136 (1999), 9-74. MR 1681113 Zbl 0910.35107 
[15] A. Soffer and M. I. Weinstein, Selection of the ground state for nonlinear Schrödinger equations. Rev. Math. Phys. 16 (2004), 977-1071. MR 2101776 Zbl 1111.81313

[16] T.-P. Tsai and H.-T. Yau, Asymptotic dynamics of nonlinear Schrödinger equations: resonance-dominated and dispersion-dominated solutions. Comm. Pure Appl. Math. 55 (2002), 153-216. MR 1865414 Zbl 1031.35137

[17] T.-P. Tsai, Asymptotic dynamics of nonlinear Schrödinger equations with many bound states. J. Differential Equations 192 (2003), 225-282. MR 1987092 Zbl 1038.35128

[18] M. I. Weinstein, Modulational stability of ground states of nonlinear Schrödinger equations. SIAM J. Math. Anal. 16 (1985), 472-491. MR 0783974 Zbl 0583.35028

Received June 24, 2012; revised October 15, 2012

Alexander Komech, Faculty of Mathematics Vienna University and Institute for the Information Transmission Problems RAS, Oskar-Morgenstern-Platz 1, 1090 Vienna, Austria

E-mail: alexander.komech@univie.ac.at

Elena Kopylova, Faculty of Mathematics Vienna University and Institute for Information Transmission Problems RAS, Oskar-Morgenstern-Platz 1, 1090 Vienna, Austria E-mail: elena.kopylova@univie.ac.at

Sergey Kopylov, Russian State University of Tourism and Service, Glavnaia, 99, 141221 Cherkizovo, Pushkin district, Moscow region, Russia

E-mail: PDEsys@gmail.com 